\title{
Psychological Impact of Acne on Women
}

\section{Ruksana Hamid ${ }^{1}$, Rayees ul Hamid Wani ${ }^{2}$, Suhail Hamid Wani ${ }^{3}$, Ghulam Mustafa Malik ${ }^{4}$ and Mohammad Sarwar Mir ${ }^{5 *}$}

${ }^{1}$ Anesthesiologist, Directorate of Health Services, Kashmir, India

${ }^{2}$ Senior Resident, Department of Emergency Medicine, SKIMS, Soura, India

${ }^{3}$ Post Graduate Scholar, Department of Radiodiagnosis, GMC Srinagar, India

${ }^{4}$ Post Graduate Scholar, Department of Pathology, GMC Srinagar, India

${ }^{5}$ Resident Medical Officer, SKIMS, Soura, India

*Corresponding Author: Mohammad Sarwar Mir, Resident Medical Officer, SKIMS, Soura, India.
Received: March 15, 2021

Published: March 24, 2021

(C) All rights are reserved by Mohammad

Sarwar Mir., et al.

\begin{abstract}
Background: It is well-known that acne vulgaris is a common malady of adolescence.

Objective: To evaluate the level of impact among acne female adolescent students on their quality of life.
\end{abstract}

Methods: A total of 200 patients studied. Acne severity was graded using Global Acne Grading System (GAGS) after the clinical diagnosis. All the patients went through self administered questionnaire of Cardiff Acne Disability Index (CADI) to fill out, to assess the reflection of patients' experiences and perceptions.

Results: The maximum number of patients was in the age group of 16 - 20 years $(142 / 200,71 \%)$. The impact on quality of life was more in the age-group of 21 - 30 years even though in this age group clinical severity of acne was mild to moderate only.

Conclusion: Study found that women with acne had profound emotional, as well as, social impact on their quality of life.

Keywords: Acne; Cardiff Acne; Disability Index; Quality of Life

\section{Introduction}

It is well-known that acne vulgaris is a common malady of adolescence and is easily recognized. It is common enough to be called as physiological process, but is better recognized disease due to its inflammatory component. It can have significant psychosocial disturbances as it affects adolescence that is the critical period during which emotional development and maturity starts taking place [1].

Skin plays an important role in socialization, as skin is considered as an organ of Communication. Cosmetic disfigurement produces social disapproval and increase in self-consciousness [2]. It can result in social poor eye contact, academic under achievements and psychosocial disturbances especially when skin diseases like acne occur during adolescence [3]. Acne may also be associated with increased levels of anxiety, anger, depression and frustration. This psychological stress can further aggravate acne through hormonal changes thereby setting up a vicious cycle. It is important to have reliable epidemiological data on acne and its psychosocial impact so that physician can show quantitatively that effective treatment of acne can have significant benefit to patient's psychosocial life, apart from physical benefit to their acne [4].

\section{Methods}

This study was a cross sectional survey. A total of 200 female patients with clinical diagnosis of acne, aged more than 13 years were enrolled in the study after taking an informed consent.

A detailed history was also taken according to Performa. The severity of acne was graded using Global Acne Grading System 
(GAGS). The patient was then given a self-administered Cardiff Acne Disability Index (CADI) questionnaire Global Acne Grading System (GAGS) Acne was graded using GAGS [5].

The GAGS considers six locations on face and chest/ upper back with a factor for each location based on surface area, distribution and density of pilosebaceous units. (Area factor: forehead-2, right cheek-2, left cheek-2, nose-1, chin-1, chest and upper back-3). Each of the location was graded separately on 0-4 scale with the most severe lesion within that area determining the local score. (Grade: 0-no lesion, 1-comedone, 2- papule, 3-pustule, 4-nodule). Under good light and without touching, all the manifestations of acne ranging from comedones to nodule were recorded. Local score is calculated by multiplying area factor with the grade of most severe lesion within that location. Global score is the summation of all the local scores.

\section{Cardiff acne disability index (CADI)}

All the enrolled patients with acne above the age of 13 years completed CADI questionnaire made available in local language. It is a detailed questionnaire designed to assess disability caused by acne [6]. It consists of five questions, each with four graded alternative responses (0-3). CADI is calculated by summing up the score of each question. The higher score indicates the greater disability experienced and the lower score indicates the lesser level of disability.

\section{Results}

Out of 200 patients, the maximum number of patients was in the age group of $16-20$ years $(142 / 200,71 \%)$. Family history of acne was present in $80(40.0 \%)$ patients. Appearance of facial acne was preceded by application of topical steroids in 40 (20\%) patients.

\begin{tabular}{|l|c|c|c|}
\hline Acne severity (GAGS) & Males & Females & Total \\
\hline Mild & 36 & 23 & 59 \\
\hline Moderate & 34 & 29 & 63 \\
\hline Severe and very severe & 16 & 62 & 78 \\
\hline Total & 86 & 114 & 200 \\
\hline
\end{tabular}

Table 1: Relationship between acne severity global acne grading system (GAGS) and gender.

$P$ value $<0.05$ (Non-Significant)

\begin{tabular}{|l|c|c|c|c|}
\hline Duration & Low & Medium & High & Total \\
\hline Less than six months & 5 & 8 & 13 & 26 \\
\hline 6 months-one year & 12 & 16 & 20 & 48 \\
\hline Greater than one year & 12 & 45 & 69 & 126 \\
\hline Total & 38 & 80 & 82 & 200 \\
\hline
\end{tabular}

Table 2: Relationship between Cardiff acne disability index (CADI) and duration of acne. $\mathrm{P}=0.001$; highly significant.

\begin{tabular}{|l|c|c|c|c|}
\hline GAGS & Low & Medium & High & Total \\
\hline Mild & 11 & 48 & 31 & 90 \\
\hline Moderate & 12 & 18 & 40 & 70 \\
\hline Severe and very severe & 3 & 12 & 25 & 40 \\
\hline Total & 26 & 78 & 96 & 200 \\
\hline
\end{tabular}

Table 3: Relationship between global acne grading system (GAGS) and cardiff acne disability index (CADI).

\section{Discussion}

Acne has shown significant psychological impact in the previous studies. The severity of acne was measured by different grading methods in various studies.

Most of the patients were adolescents as acne is more prevalent in this age group [7].

About $40.0 \%$ of patients gave history of acne in parents or siblings. This is in accordance with various studies which show that genetic factors influence susceptibility to acne [8]. One-fifth of patients had applied topical steroids which lead to further aggravation of acne. It is due to easy availability and indiscriminate use of topical steroids and marked transient improvement which ultimately results in steroid dependence.

Acne may have different psychosocial morbidity according to gender. Our study showed higher CADI scores in females. This is similar to previous studies which also found more psychological impact on females [9]. Facial disfigurement makes females present earlier to treatment even for milder acne. The impact on quality of life was more in the age-group of 21-30 years even though in this age group clinical severity of acne was mild to moderate only. It may be due to chronicity of acne and the need of more social inter- 
actions at this age. All the patients above 30 years of age had low psychological impact of acne.

Patients with persistent acne were reported to have higher CADI scores [9]. This study demonstrated a significant relation between GAGS and CADI scores. The impact on quality of life increased with acne severity. It is consistent with previous studies which also found the similar findings [9-11]. Also patients with higher CADI scores tend to present with suicidal ideations.

\section{Recommendations}

In order to decrease psychological stress associated with acne and to build up self-esteem, it is the need of hour in a developing country like India to establish acne clinic with provision of counselors. This need stems out of high patient burden with low doctor: patient ratio. In order to increase time spent per patient, these acne clinics with counselors will help achieve the targets. Also the patients can be involved in group discussions.

\section{Conclusion}

Our study confirmed that acne has profound effect on psychosocial behaviour. The impact on quality of life has been significantly associated with severity of acne, female gender, age and long disease duration. Clinicians should be aware that early treatment of acne and avoidance of aggravating factors can prevent significant psychological morbidity.

\section{Conflict of Interest}

None.

\section{Bibliography}

1. Mubashar MM and Mohammad S. "Psychosocial Impact of Acne: A Community Based Cross Sectional Study". Journal of Psychology and Psychotherapy Research 1.3 (2018).

2. Koo J. "The psychosocial impact of acne: patients' perceptions". Journal of the American Academy of Dermatology 32 (1995): S26-S30.

3. Fried RG and Wechsler A. "Psychological problems in the acne patient”. Dermatology Therapy 19 (2006): 237-240.

4. Thomas DR. "Psychosocial effects of acne". Journal of Cutaneous Medicine and Surgery 8 (2004): 3-5.
5. Doshi A., et al. "A comparison of current acne grading systems and proposal of a novel system". International Journal of Dermatology 36 (1997): 416-418.

6. Motley RJ and Finlay AY. "Practical use of a disability index in the routine management of acne". Clinical and Experimental Dermatology 17 (1992): 1-3.

7. Burton JL., et al. "The prevalence of acne vulgaris in adolescents". British Journal of Dermatology 197185 (1971): 119126.

8. Walton S., et al. "Genetic control of sebum excretion and acne. A twin study". British Journal of Dermatology 18 (1988): 393396.

9. Do JE., et al. "Psychosocial Aspects of Acne Vulgaris: A Community-based Study with Korean Adolescents". Annals of Dermatology 21 (2009): 125-129.

10. Atkan S., et al. "Anxiety, depression and nature of acne vulgaris in adolescents". International Journal of Dermatology 39 (2000): 354-357.

11. Hanisah A., et al. "Prevalence of acne and its impact on the quality of life in school-aged adolescents in Malaysia". Journal of Primary Health Care 1 (2009): 20-25.

\section{Assets from publication with us}

- Prompt Acknowledgement after receiving the article

- Thorough Double blinded peer review

- Rapid Publication

- Issue of Publication Certificate

- High visibility of your Published work

Website: www.actascientific.com/

Submit Article: www.actascientific.com/submission.php Email us: editor@actascientific.com

Contact us: +919182824667 